Jochen Strobel

\title{
Kollaborative Strukturen in der digitalen Edition. Akteure, Rollen, Verantwortlich- keiten, Rechtliches
}

Wissenschaftliche Editionen kennen auch in der Gutenberg-Galaxis eine Vielzahl von Rollen und Verantwortlichkeiten, die von Projekt zu Projekt neu definiert werden und insgesamt auf wandelbare, oft implizit bleibende Konventionen zurückzuführen sind. Dabei ist zwischen dieser sich etwa auf dem Titelblatt einer Edition manifestierenden Konventionalität und den Anforderungen des Urheberrechts immer schon zu unterscheiden. Nun steht zu vermuten, dass digitale Editionen die Praxis der Zusammenarbeit verändern bis verkomplizieren und dass infolgedessen neben vielen anderen Parametern auch die Definitionen von ,Akteuren' und ,Rollen' revidiert und erweitert werden müssen. Neben einer hochgradig bedeutsam gewordenen technischen Komponente sind es die Infrastrukturen und personenbezogenen Aufgaben der Nachhaltigkeit sowie die möglich und notwendig gewordenen Strukturen der institutionellen, personellen und datenspezifischen Vernetzung und Standardisierung, die viel stärker als bisher in Erwägung gezogen werden müssen. Digitale Editionen kommen anders zustande als die gewohnten Printausgaben, sie erfordern von allen beteiligten Personen und Institutionen neue Praktiken, sie verändern Projektziele und -ergebnisse und sie nehmen auch Einfluss auf die daran beteiligten Akteure. Sie lassen, mit anderen Worten, andere Wissenschaftler`innen und weitere Beteiligte entstehen als bisher.

Es gilt im Folgenden, Beobachtungen wiederzugeben und $\mathrm{zu}$ analysieren sowie Vorschläge für eine künftige Praxis zur Diskussion zu stellen. Neben den rechtlichen Implikationen (Urheberrecht, Lizenzmodelle, Projektverantwortlichkeiten) sind es die Karriereoptionen der Beteiligten, die in den Blick genommen werden müssen. Dass das Internet nicht anonym ist, Projekt-Homepages die Namen der Verantwortlichen mehr oder weniger versteckt nennen, tröstet Wissenschaftler*innen, die ihr Projekt etwa vergeblich in Bibliothekskatalogen suchen (und es dann en passant im Datenbank-Infosystem ihrer Universitätsbibliothek finden ${ }^{1}$ ), nur wenig.

1 Vgl. aus dem Blickwinkel des Angehörigen der Universität Marburg: http://rzblx10.uni-regensburg.de/dbinfo/fachliste.php?bib_id=ubma\&lett=1\&colors=\&ocolors= (31.1.2018).

ว Open Access. (c) 2018 Jochen Strobel, publiziert von De Gruyter. (ध) BY-Nc-ND Dieses Werk ist lizenziert unter der Creative Commons Attribution-NonCommercial-NoDerivatives 4.0 Lizenz. 
Die 2017 von Fotis Jannidis u. a. herausgegebene Einführung in die Digital Humanities enthält erfreulicherweise ein Kapitel „Rechtsfragen digitaler Geistes- und Kulturwissenschaft“, das den Akteuren Sensibilität für rechtliche Fragen auf die Fahnen schreibt. Die wichtige, im jeweiligen CC-Lizenzmodell ${ }^{2}$ sich manifestierende Entscheidung über die Modalitäten der Nachnutzbarkeit in Open Access verfügbarer editorischer Daten setzt im Grunde eine andere Entscheidung voraus, nämlich

dass alle im Rahmen von Projekten bei den Beteiligten entstehenden Urheber- und Leistungsschutzrechte unter einheitlichen und offenen Bedingungen bei der Projektleitung bzw. bei der das Projekt tragenden Institution gebündelt werden, um eine möglichst einfache und weitreichende Nachnutzbarkeit der Projektergebnisse zu garantieren. ${ }^{3}$

Urheber streben jedoch nicht nur möglichst weitreichende Nutzung des von ihnen Geschaffenen gewissermaßen um jeden Preis an, sondern sind an angemessener Wertschätzung und Wertschöpfung interessiert. Wertschätzung bedeutet, dass nicht in erster Linie Namen von Institutionen, sondern Namen von Personen mit Projektergebnissen in Verbindung gebracht werden können. Dennoch kommt den Institutionen langfristig die entscheidende Rolle $\mathrm{zu}$, müssen diese also auch entsprechend in der Edition genannt werden.

Patrick Sahle sieht in der digitalen Edition einen Nährboden für kollaboratives Arbeiten in verteilten Rollen in Teamwork:

Eine idealtypische Gliederung sieht den ,general editor vor allem als Leiter und Moderator verschiedener Gruppen von Zuarbeitern. In einer idealtypischen Beschreibung können solche Editionsteams dann z.B. nach folgenden Rollen und Aufgaben beschrieben werden:

- Leitung der Gesamtedition, Kommunikation, Außenvertretung

- Beratung zu Fragen der Überlieferung, der einzusetzenden Technik, der grundlegenden editorischen Konzepte, der konkret anzuwendenden Methoden und der Publikationsformen

- Grunderschließung der Überlieferung

- Bilddigitalisierung und Qualitätskontrolle

- Textgewinnung, Transkription

- Vertiefende Texterschließung, inhaltliche und philologische Kritik, wissenschaftliche Annotation

- Datenmanagement (Entwurf der Datenstrukturen, Konversionen)

- Serveradministration (bei Online-Editionen)

- Konzeptionierung der Publikationsformen. ${ }^{4}$

2 Vgl. https://de.creativecommons.org/ (31.1.2018).

3 Steinhauer, Eric: Recht. In: Digital Humanities. Eine Einführung. Hrsg. von Fotis Jannidis, Hubertus Kohle u. Malte Rehbein. Stuttgart: Metzler 2017. S. 345-352, hier S. 351. 
Derart ausdifferenzierte Aufgabenspektren wird es nur in sehr großen Projekten geben können. Anne Baillot und Thomas Ernst fächern in ihrem Text Was kennzeichnet die digitale wissenschaftliche Autorschaft? dieses Rollenspektrum teils etwas anders, teils auch noch weiter auf, mit Rollen wie „Moderator/in; [...] Mitdiskutant/in, [...] Annotator/in, Tagger/in; [...] wissenschaftliche Hilfskraft; Lizensor/in, [...] Crowdfunder/in, Sponsor/in, Crowdsourcer/in. “5

Fürwahr: eine immense Zahl an möglichen Mitspieler`innen in teils mehreren Rollen ist zu erwarten. Indessen sind manche dieser Rollen nicht so neu, Juristen oder Hilfskräfte sind nur oft nicht sonderlich hervorgetreten, d. h. sie bleiben traditionell ungenannt. Daher ist es zu begrüßen, wenn Baillot und Ernst anmahnen, die diversen Rollen sollten „im Kontext des jeweiligen wissenschaftlichen Beitrags differenziert und klar zugeschrieben werden“, beispielsweise in Gestalt von Versionierungen. ${ }^{6}$ Die im zitierten Artikel empfohlene, von Autor/Name nach Kurztitel/Version umstellende Zitierweise nimmt aber die Emphase um die Autor^innenrollen denkbar weit zurück, indem Personennamen verschwinden. Auch die Vorstellung, digitale Editionen entstünden künftig vor allem dank ,Schwarm' oder ,Crowd', unterschätzt, dass schon aus arbeitsrechtlichen Gründen und aus solchen der Projektverantwortlichkeit Namen in den gebotenen Abstufungen genannt werden müssen. Festzuhalten ist vielmehr mit Sahle, dass im vielfach verzweigten Arbeiten tatkräftige Projektleitung (also so detailliert wie möglich: Projektmanagement), wissenschaftliche Verantwortlichkeit und nach Möglichkeit auch rechtsverbindliche Verantwortlichkeit (Herausgeberschaft) zusammengefasst sein sollten. ${ }^{7}$ Nicht Machtfülle, sondern collaborare steckt als Zielsetzung dahinter. Es möge keinen Verantwortlichen und durch das Urheberrecht Geschützten geben, der faktisch nicht mitarbeitet.

Das Urheberrecht kennzeichnet Autorschaft und wissenschaftliche Herausgeberschaft unisono als Urheberschaft, unterscheidet also nicht dem Begriff nach zwischen dem Autor als gewissermaßen ,eigentlichem' Schöpfer und dem-

\footnotetext{
4 Sahle, Patrick: Digitale Editionsformen. Zum Umgang mit der Überlieferung unter den Bedingungen des Medienwandels. Bd. 2: Befunde, Theorie und Methodik. Norderstedt: Books on Demand 2013, S. 231.

5 Baillot, Anne u. Thomas Ernst: Was kennzeichnet die digitale wissenschaftliche Autorschaft? http://dhd-wp.hab.de/?q=content/2-was-kennzeichnet-die-digitale-wissenschaftliche-autorschaft (31.1.2018).

6 Baillot/Ernst, Was kennzeichnet (wie Anm. 5).

7 Baillot/Ernst nennen als Beispielgruppe für kollaboratives digitales wissenschaftliches Arbeiten Wikis - doch kann kein Wissenschaftler seine berufliche Laufbahn auf kollektiv und anonym entstandene Onlineartikel in Wikis gründen. Anonymität und Pseudonymität vertragen sich vielmehr mit wissenschaftlichem Arbeiten nur sehr begrenzt, bedeutet dieses doch persönliches Zeugnis von seinem Denken abzulegen, sich gewissermaßen mit offenem Visier einer Debatte auszusetzen.
} 
jenigen, der nur die von jemand anderem geschaffenen Werke herausgibt. Daher heißt es in $\S 70 \mathrm{im} 2$. Teil des UrhG:

Ausgaben urheberrechtlich nicht geschützter Werke oder Texte werden in entsprechender Anwendung der Vorschriften des Teils 1 geschützt, wenn sie das Ergebnis wissenschaftlich sichtender Tätigkeit darstellen und sich wesentlich von den bisher bekannten Ausgaben der Werke oder Texte unterscheiden.

(2) Das Recht steht dem Verfasser der Ausgabe zu. ${ }^{8}$

Dieser Schutz endet aber bereits nach 25 Jahren, nicht wie der von Werken auf Schöpfungshöhe 70 Jahre nach dem Tod des Verfassers. Also nicht dem Begriff nach, aber der Dauer des rechtlichen Schutzes nach trifft das Gesetz eine Unterscheidung.

\section{II}

Ausgerechnet die Literaturwissenschaft hat sich ein hehres Bild von Herausgeberschaft bewahrt, wohl nicht nur weil die Aufgabe, aufbereitete Quellen einer wissenschaftlichen Community zur Verfügung zu stellen, zu den das Fach oder besser Fächerspektrum begründenden Tätigkeiten gehört, sondern auch weil diese Quellen selbst Begriffe und Praktiken des Verfassens und des Herausgebens umkreisen. Als sich im 18. Jahrhundert, beispielsweise um den Begriff des Genies herum, bald aber auch um die Frage des ,Besitzes‘ von Werken, die Funktion ,Autor ' herauszubilden begann, ${ }^{9}$ begleitete die Fiktionalisierung, also das Authentizitätsversprechen von Autorschaft im fiktionalen Text, eine rahmende Funktion ,Herausgeber‘. Etwa als der Sammler, Arrangeur und Kommentator von angeblich gefundenen Briefen oder Manuskripten tritt eine ebenso fiktive Instanz namens Herausgeber auf, der den Autor gewissermaßen erst ,macht‘, seinen Text beglaubigt und dessen Anteile am Text selbst (eben: Sammeln, Auswählen, Weglassen, Verändern, Kommentieren ...) paradigmatisch unklar bleiben. Der Herausgeber als „Dazuschreiber“, ${ }^{10}$ so der Gießener Literaturwissenschaftler Uwe Wirth, sichert das Prinzip einer Einheit des Schreibens. In der Genie-Epoche steht also die Vorstellung originärer und gewissermaßen

\footnotetext{
8 https://www.gesetze-im-internet.de/urhg/UrhG.pdf (31.1.2018).

9 Klassisch hierzu: Foucault, Michel: Was ist ein Autor? In: Michel Foucault: Schriften zur Literatur. Frankfurt am Main: Fischer 1988. S. 7-31.

10 Wirth, Uwe: Die Geburt des Autors aus dem Geist der Herausgeberfiktion. Editoriale Rahmung im Roman um 1800. Wieland, Goethe, Brentano, Jean Paul und E. T. A. Hoffmann. Paderborn: Fink 2008, S. 15.
} 
auch einsamer Hervorbringung neben der einer kollektiven, eben eines Dazuschreibens durch Zweite und Dritte.

Wie sehr auch immer die Romantiker Leser zu weiteren oder erweiterten Autoren ernennen mochten, wie sehr sie auch der Idee kollektiver Autorschaft aus dem Symphilosophieren heraus anhängen mochten: collaborare war eine ihrer größten Tugenden. Neben dem Übersetzen oder dem Nachdichten pflegten sie vielfach auch die (gemeinsame) Herausgeberschaft. Dies gilt gleichermaßen für die Weimarer Konkurrenz: Der alte Goethe beispielsweise sammelte nicht nur seine Briefwechsel mit den Freunden Friedrich Schiller und Carl Friedrich Zelter, er schrieb nicht allein den mit Carl Friedrich Zelter buchstäblich bis in seine letzten Tage hinein fort, er sammelte eingegangene Briefe, Abschriften und auch die von ihm abgesandten Originale; er agierte schließlich als Herausgeber post (bei Schiller) und prae mortem (bei Zelter). Die so entstandenen Briefeditionen ließen eine Unterscheidung zwischen Autorschaft und Herausgeberschaft nicht zu.

Die edierenden Philologen seit Karl Lachmann legten berechtigterweise Wert darauf, dass ihre Rolle und Funktion transparent würde. Jüngere, teils in großen Teams entstandene Editionen haben diese Transparenz in vielfacher Abstufung als Titelblatt-Philologie akribisch und ehrgeizig fortgesetzt. So nennt ein Band der neunten Abteilung der Kritischen Gesamtausgabe von Nietzsches Werken, an dem der Verfasser mitwirkte, insgesamt 18 Personennamen und eine Institution, die in insgesamt sieben Kategorien aufgeschlüsselt werden. Versteht man die jeweilige Zuschreibung zu lesen, dann kann man die Rollen vom bereits verstorbenen Begründer über die Reihenherausgeber, den Projektverantwortlichen, die Teamleiterin, den Bearbeiter bis zur mitwirkenden studentischen Hilfskraft unterscheiden. ${ }^{11}$ Über diese Ausführlichkeit lässt sich streiten, für den wissenschaftlichen Nutzer ist sie allemal aussagekräftig. Zum eingeführten Begriffs- und Abstufungssystem gehören etwa Reihen-, Band(mit)herausgeberschaft vs. Bandbearbeiterschaft, Zusammenarbeit, Mitarbeit und weitere nur diskursintern aussagekräftige Formulierungen wie „in Verbindung mit“ oder „gemeinsam mit“.

Digitale Editionen scheinen nun, wie im Internet üblich und wie in Zitierpraktiken nach Internetquellen leider häufig, Namensnennungen klein(er) zu schreiben. Es sind die beteiligten Institutionen und die Förderer, die etwa auf der Startseite des Heinrich-Heine-Portals genannt werden. ${ }^{12}$ Sodann finden sich mehrere Listen der Mitarbeiter`innen mit ihren jeweiligen Herkunftsinstitutio-

11 Nietzsche Werke. Kritische Gesamtausgabe. Begründet von Giorgio Colli u. Mazzino Montinari. Neunte Abteilung, Vierter Band. Berlin, New York: de Gruyter 2004.

12 http://www.hhp.uni-trier.de/ (31.1.2018). 
nen, nicht aber mit ihren konkreten Funktionen. ${ }^{13}$ Eine verantwortliche Herausgeberschaft scheint es nicht zu geben. Dies ist nicht ungewöhnlich, da das Portal vorwiegend auf Retrodigitalisierung beruht - allerdings medientypische Funktionalitäten und zusätzliche Texte und Metadaten bereithält! Die entstehenden digitalen Editionen der Werke Carl Maria von Webers ${ }^{14}$ oder der Notizbücher Theodor Fontanes ${ }^{15}$ nennen Projektmitarbeitende wie -verantwortliche mit klarer Unterscheidung der jeweiligen Funktion. Im Falle Fontanes wird die Herausgeberin auch im Zitiervorschlag genannt. ${ }^{16}$ Die Herausgeberin und editorische Hauptmitarbeiterin Gabriele Radecke ist zugleich Projektleiterin die Übernahme mehrfacher Funktionen muss nicht grundsätzlich der Fall sein, kann es aber in kleineren Projekten durchaus. Und alle drei Rollen implizieren unterschiedliche Tätigkeiten mit je unterschiedlicher Wertigkeit, etwa als Antragstellerin, Urheberrechtsbegünstigte und als Entscheidungsträgerin im Alltagsgeschäft.

\section{III}

Die von Thomas Bürger zusammen mit dem Verfasser konzipierte und seit 2012 als DFG-Projekt von beiden geleitete digitale Edition der Korrespondenz August Wilhelm Schlegels ist als Beta-Version seit 2014 online. Damit kommt eine neben der Aufnahme bereits gedruckter Briefe zweite Besonderheit des Projekts zum Tragen, nämlich eine konsequente Open-Access-Politik noch während der Projektentstehung. In den etwa sechs Jahren seit Projektbeginn haben neben den Projektleitern und dem Kooperationspartner Thomas Burch (vom Trier Center for Digital Humanities) zahlreiche Personen zum Zustandekommen der Edition beigetragen: Editorinnen, Programmierer (in Dresden und in Trier), Bibliothekar^innen, wissenschaftliche und studentische Hilfskräfte, Freunde und Bekannte, nicht zuletzt befreundete und assoziierte Wissenschaftler`innen. Ganz selbstverständlich bringen in Eigenleistung der Institutionen tätige Bibliothekare und Informatiker in Dresden und in Trier immer wieder ihre spezifi-

13 http://www.hhp.uni-trier.de/Projekte/HHP/kontakt (31.1.2018).

14 http://weber-gesamtausgabe.de/de/Projekt/Projektbeschreibung.html (31.1.2018). Gesamtherausgeber ist Gerhard Allroggen.

15 https://fontane-nb.dariah.eu/index.html (31.1.2018). Erfreulicherweise deutlich wird Gabriele Radecke als Herausgeberin bereits auf der Startseite genannt.

16 https://fontane-nb.dariah.eu/content.html?id=zitationshinweise.md (31.1.2018). Das Editionsteam wird auf einer Seite ausführlich vorgestellt: https://fontane-nb.dariah.eu/content. html?id=editionsteam.md (31.1.2018). 
schen Kompetenzen in das Projekt ein. Die Universität Marburg trägt vor allem fachwissenschaftlich-editorische Arbeit bei. Das Impressum bietet uns die Chance, alle Beteiligten auf faire Weise zu nennen und ihre Rollen zu beschreiben. Letzteres mag noch ausbaufähig sein; die Trennung zwischen aktuellem Team ${ }^{17}$ und der Summe der jemals Mitarbeitenden ${ }^{18}$ scheint uns aber angezeigt. Spätestens mit dem Versionswechsel von Beta auf „Version 1.0“, die für den 1. Oktober 2018 geplant ist, werden der Verfasser zusammen mit der wissenschaftlichen Mitarbeiterin Claudia Bamberg als Herausgeber genannt werden. Dies gilt analog bereits jetzt für die XML-TEI-Daten unserer Edition. Projektbezeichnungen und Versionen helfen auch den Nutzern, die Grenzen des Projekts einschätzen zu können, also Beginn, Abschluss und nach Möglichkeit eine Projektion in die Zukunft nach dem offiziellen Projektabschluss. Herausgeberschaft ad personam impliziert die unbegrenzte Dauer der Verantwortlichkeit gegenüber den Projektdaten. Für die Zuständigkeit der Institution umso mehr, aber je nach Kompetenz der beteiligten Institution.

Anders als die in der Trierer Forschungsumgebung FuD oder anderen Speicherorten aufbewahrten Daten unserer Edition und auch anders als die gegenwärtige Präsentationsoberfläche wird das TEI unsere Projektlaufzeit überdauern. Die TEI-Daten werden langfristig unsere Projektarbeit repräsentieren. Die Diskussion um Akteure, Rollen und rechtliche Implikationen muss also letztlich in Fragen zur Ausgestaltung des TEI-Headers münden.

\section{IV}

Für die Festschreibung der Rollen ist, wie gesagt, das Urheberrecht entscheidend. Über den schöpferischen Charakter von Editionen muss an dieser Stelle nicht weiter räsoniert werden, ihn zu demonstrieren ist eine (jederzeit mühelos zu leistende) Pflichtübung. Das Urheberrecht spricht ohnehin unter anderem bei Werkausgaben abschwächend vom Leistungsschutzrecht: ${ }^{19}$ „Belohnt wird nicht das bloße Auffinden eines verschollenen Textes, sondern die wissenschaftliche, textkritische Leistung “. ${ }^{20}$ Leistungsschutz impliziert hier vielleicht weniger Wertschöpfung als Schutz vor Plagiaten in Anbetracht von Open Ac-

$17 \mathrm{http}: / /$ www.august-wilhelm-schlegel.de/briefedigital/contact (31.1.2018).

$18 \mathrm{http} / / /$ www.august-wilhelm-schlegel.de/briefedigital/sitenotice (31.1.2018).

19 Vgl. etwa: Seyavash, Amini: Digitale Kultur zum Pauschaltarif? Anlass, Inhalt und Grenzen einer Vision für das Urheberrecht der Zukunft. Baden-Baden: Nomos u. a. 2017, S. 53-55.

20 Schack, Haimo: Urheber- und Urhebervertragsrecht. 8., neu bearbeitete Aufl. Tübingen: Mohr Siebeck 2017, S. 381. 
cess. Die informatische Urheberschaft an auf Anweisung eines Arbeitgebers hergestellten Datenbankwerken wird durch das Urheberrecht zurückhaltend bewertet: „Der Arbeitgeber kann regelmäßig von einem umfassenden Erwerb der ausschließlichen Nutzungsrechte ausgehen.“21

Das Problem der Beteiligung ist in der Rechtsprechung ausführlich diskutiert worden. Gemeinsame Urheberschaft impliziert die Individualität eines Mitwirkenden, die im Werk zum Ausdruck kommen muss, sofern auch tatsächlich ein gemeinsames Werk entstanden ist. ${ }^{22}$ „Die Miturheberschaft setzt ferner auch eine Zusammenarbeit in der Weise voraus, dass sich die Beteiligten über die gemeinsame Aufgabe verständigen und sich gegenseitig unter die Gesamtidee unterordnen." 23 Im Übrigen ist die Rechtsstellung des wissenschaftlichen Urhebers unter anderem davon abhängig, ,welche Werke in Erfüllung der arbeitsvertraglichen oder dienstvertraglichen Verpflichtungen geschaffen werden“. ${ }^{24}$

Wie die Abgrenzung der Herausgeberschaft von weiteren Rollen aussieht, demonstriert die Verwertungsgemeinschaft Wort (VG Wort) mit ihrer Verwaltungspraxis. Editorische Kommentierung als Schöpfung im Rahmen wissenschaftlicher Ausgaben wird von der VG Wort akzeptiert. Doch werden nur die Herausgeber beteiligt, nicht etwa ,Bearbeiter' oder ,Mitarbeiter', bzw. dies geschieht nur, sofern die Herausgeber dem zustimmen. ${ }^{25}$ Der Verteilungsplan für

21 Lührig, Nicolas. In: Handbuch Urheberrecht und Internet. Hrsg. von Jürgen Ensthaler u. Stefan Weidert. 3., überarbeitete u. erweiterte Aufl. Frankfurt am Main: Deutscher Fachverlag GmbH, Fachmedien Recht und Wirtschaft 2017, S. 121.

22 Vgl. Bisges. In: Handbuch Urheberrecht. Hrsg. von Marcel Bisges. Berlin: Erich Schmidt Verlag 2016, C. Entstehung des Urheberrechts und Urheberschaft, Abschnitte 310f.

23 Bisges (wie Anm. 22), Abschnitt 312.

24 Moor, André: Rechtsstellung des wissenschaftlichen Personals im Urheberrecht. Diss. Münster 2017, S. 163. Beachtenswert oder vielleicht besser kurios zu nennen ist hierbei, dass allein Hochschullehrern die nähere Ausgestaltung des Dienstverhältnisses obliegt, auch im Hinblick auf urheberrechtlich relevante Forschung, hingegen von wissenschaftlichen Mitarbeitern wissenschaftliche Dienstleistungen und nur in Ausnahmefällen eigene Forschung erwartet werden (Vgl. Moor, S. 21f.).

25 Vgl. die E-Mail von Dr. Gabriele Lotz von der VG Wort an den Verfasser vom 26. Januar 2018: „Redaktionelle Tätigkeit, Lektorat fällt nicht unter Urheberschaft und bei Angaben wie ,unter Mitwirkung، oder ,unter Mitarbeit' machen wir es von der Zustimmung desjenigen abhängig, der als Autor, Herausgeber oder Übersetzer genannt wird, ob er die so Bezeichneten als gleichwertige Miturheber ansieht. Eine Reihenherausgabe oder die Herausgabe von Zeitschriften kann nicht gemeldet oder vergütet werden.“ (Ich danke Frau Dr. Lotz herzlich für ihre Auskunft.) 
Ausschüttungen ab $2018^{26}$ sieht in $\S 3$, Satz 6 vor: „Herausgeber einer wissenschaftlich kommentierten Ausgabe eines urheberrechtlich geschützten Werks werden mit $25 \%$ [des ausschüttungsfähigen Anteils] berücksichtigt.“ Bei Onlinepublikationen entscheiden Zugriffszahlen über die Ausschüttung. ${ }^{27}$

\section{V}

Dass urheberrechtlich durch editorische Herausgeberschaft Begünstigte selbst auch intellektuellen Nutzen aus den erarbeiteten Daten ziehen, sollte selbstverständlich sein. Zur Mitarbeit im Projektteam in konzeptioneller Hinsicht, möglichst aber auch in Gestalt eines Abgleichs aller freizuschaltenden Daten, sollte für die Herausgeber, ebenfalls zusammen mit interessierten Teammitgliedern, die weitere wissenschaftliche Arbeit mit der Edition stehen, freilich mit Schwerpunktsetzungen, die den Forschungsinteressen entsprechen. Herausgeberschaft impliziert auch die Anregung und Durchführung von Tagungen, die Konzeption von Sammelbänden sowie die Nutzbarmachung von Projektinhalten in der universitären Lehre, in Hilfskraftstellen, Abschlussarbeiten und ebenso in Grenzbereichen wie Praktika. Dies alles gehört seit langer Zeit zum Alltag universitärer Verschränkung von Lehre und Forschung - doch zu den weiteren Stärken kollaborativer und vernetzter Arbeit in digitalen Editionsprojekten gehören eben auch diese traditionell anmutenden Anknüpfungspunkte. Sie müssen dennoch genannt werden, da neue Projektrollen zugleich neue Interessen und Funktionen in Lehre und Forschung bedeuten: Die zwischen Fachwissenschaftler und Informatiker diskutierten Visualisierungen könnten in die Lehre einfließen, studentische Abschlussarbeiten wiederum mögen sich theoretisch und praktisch-exemplarisch mit den Essentials digitaler Edition befassen. Beteiligte ITMitarbeiter ${ }^{\star}$ innen könnten sich mit ihrer Sicht auf Projektdaten in wissenschaftlichen Beiträgen zu Wort melden. Schließlich könnte Crowdsourcing zu kolloquiumsartigen Nutzertreffen und $\mathrm{zu}$ wissenschaftlicher Thesenbildung führen und umgekehrt. Ist die Edition selbst eine ,Schöpfung‘? Wo aber beginnt, wo endet die Edition? Mit den Links zu Metadaten oder zu anderen Projekten oder beispielsweise schon mit der dokumentierten Textkritik? Ist das Taggen, dem oft ausgiebige Recherchen vorauslagen, eine Schöpfung, entstehen hier Forschungsdaten? Sind Übergänge zwischen digitalen Registern und Thesauri einerseits, Transkriptionen und Briefdatierungen, ausführlichen Kommentaren

26 http://www.vgwort.de/fileadmin/pdf/verteilungsplan/Verteilungsplan_2017__fuer_Ausschuettungen_ab_2018_.pdf (31.1.2018).

27 Vgl. § 52, Abs. 1 und 2. 
und Einführungstexten andererseits denkbar, die die zumindest gefühlten Grenzen zwischen Datenerhebung und ,Edition als Schöpfung hinter sich lassen?

Eine grobe Unterscheidung ist die schon hergeleitete zwischen Herausgeber`in und weiteren Beteiligten, gelte diese Beteiligung für einzelne Datensätze oder für das Gesamtprojekt. Um beides zu dokumentieren, muss das TEI jedes Datensatzes (in unserem Projekt etwa: je Brief) einen Gesamtheader enthalten und einen datensatzspezifischen Header. Auch der Header folgt einer Konvention oder könnte einer solchen folgen. Die Arbeit der TEI-SIG Correspondence ist dokumentiert. ${ }^{28}$ Unter anderem haben sich die deutschen Mitglieder Stefan Dumont und Sabine Seifert zwischen 2008 und 2014 an dieser SIG beteiligt. Es handelt sich offenbar um einen ongoing process, nicht um ein festes, abgeschlossenes Regelwerk. Es ist bekannt, dass sich das TEI von Projekt zu Projekt unterscheidet. Doch auch hier müssen Konventionen überdacht, müssen Standards begründet und ausgeprägt werden.

Im Header müssen Akteure, Institutionen, Rollen, Verantwortlichkeiten möglichst transparent, trennscharf und dem deutschen Urheberrecht entsprechend festgehalten werden. In erster Linie sind es Verantwortlichkeiten und damit Personennamen, in zweiter Linie auch Namen von Institutionen, die zu nennen sind. Dies betrifft das Projekt insgesamt und zugleich den einzelnen Datensatz. In dritter Linie müssen, möglichst in nachvollziehbaren Abstufungen, die Namen aller am jeweiligen Datensatz Beteiligten gelistet werden, die so genannten collaborators.

Die Zweiteilung des Headers verdeutlicht, dass es nur noch zwei Größenordnungen produzierter und publizierter Daten gibt: Gesamtprojekt und Einzeldatensatz. Dies zu unterstreichen ist von Bedeutung, da Zwischenphänomene wie ,Bandherausgeberschaft' oder ,Bearbeitung des Kommentars‘ nicht mehr denkbar sind. Allerdings kann auf Zuständigkeiten für einzelne Register oder sonstige Thesauri hingewiesen werden.

Verantwortlich im rechtlichen Sinn sind für die Durchführung wie auch für die Ergebnisse von Forschungsprojekten zunächst die Projektleiter, im Zeitalter von Forschungsdaten in zweiter Linie (und zwar auf die lange Sicht) ihre Institutionen. Dass unsere Institutionen uns zumindest die Chance einer Datenpflege und Langzeitarchivierung einräumen, ist zu begrüßen, nicht zu perhorreszieren. Das UrhG kennt, wie ausgeführt, den Verfasser wissenschaftlicher Ausgaben als Urheber. Das entsprechende Verwertungsrecht wird berechtigterweise für Editionen inklusive Datenbankwerken reklamiert.

28 http://www.tei-c.org/Activities/SIG/Correspondence/ (31.1.2018). Den Vorschlag für einen Header finden wir hier: https://wiki.tei-c.org/index.php/SIG:Correspondence/ODD_work (31.1.2018). 
Namensnennungen haben in den Geisteswissenschaften einen anderen Stellenwert als im Bibliothekswesen und mutmaßlich auch im IT-Bereich. Für den Geisteswissenschaftler kann es der Karriere förderlich sein, wenn er als (Mit-)Autor und/oder Herausgeber, aber auch wenn er als Bearbeitender oder Mitarbeitender genannt wird. Es gilt als billig, langfristige Projektmitarbeitende, die am schöpferischen Prozess teilhaben, auch wenn sie etwa an der Projektidee nicht beteiligt waren und nicht projektverantwortlich sind, in die Herausgeberschaft einzubeziehen.

Der TEI-Header sollte rechtliche Zuständigkeiten in allererster Linie abbilden, sodann aber - unabhängig von etwaigen Konventionen - Beteiligungen namentlich nennen. Die Entscheidung muss nach eingehender Diskussion durch die Projektverantwortlichen getroffen werden. Begriffe wie Herausgeber`in, IT-Betreuer`in, Bearbeiter^in sollten fallen. Dabei sei unterstrichen, dass langjährige und für die informationstechnische Seite ausschlaggebende Mitarbeiter^innen ebenfalls namentlich zusammen mit ihrer Funktion genannt werden. Analog sollten unter orgName alle am Projekt beteiligten Institutionen genannt werden. Die collaborators werden nicht im übergeordneten ProjektHeader, sondern im Header zum Einzeldatensatz aufgeführt, am besten mit deutschsprachigen Funktionsbegriffen, die aus der Projektpraxis kommen und dem Leser mutmaßlich bekannt sein dürften, etwa: editorische Bearbeitung, Mitwirkung.

\section{VI}

Im Lichte des Urheberrechts werden sich die an digitalen Editionen mit Retrodigitalisierungsanteilen Beteiligten fragen müssen, wie sich die Herausgeberschaft von eingearbeiteten älteren Texten zur eigenen Herausgeberschaft verhält - wurden diese Texte verändert (etwa um Fehler bereinigt)? Wurden ihre Grenzen aufgehoben, indem sie durch zum Beispiel neu transkribierte Passagen ergänzt wurden? Welchen Stellenwert haben die Kommentare früherer Herausgeber für das Tagging oder auch für die Korrektur von Metadaten der ,eigenen Edition? Der ,neue' Herausgeber wird zum Meta-Editor, wenn er nach der Uneinheitlichkeit inkorporierter älterer Daten fragt, etwa sich zur Rekonstruktion einstiger, oft implizit gebliebener Editionsgrundsätze genötigt sieht.

Hinter diesen Fragen stehen immer und immer wieder Überlegungen zur wissenschaftlichen Kreativität von Editionen, die wissenschaftlichem Publizieren und mehr noch der Reklamierung von Herausgeberschaft zugrunde liegen muss. Ein Blick in die Zukunft darf nicht fehlen: Datenfriedhöfe verhindern, 
nachhaltige Projektstrukturen schaffen, die auch langfristige Weiterbearbeitung der Daten digitaler Forschungsprojekte zumindest ermöglichen - diese Aufgaben sind mit Rollendefinitionen und Verantwortlichkeiten verbunden, die über öffentlich finanzierte Projektlaufzeiten hinausgehen. Anonymität wäre als eine der Sackgassen digitalen Edierens zu bezeichnen, die Benennung von Verantwortlichkeiten und damit konkret auch die veröffentlichte Nennung von Namen gibt den an der Edition Beteiligten Sicherheit wie auch den Nutzer* innen. 